\title{
Fully portable and wireless universal brain-machine interfaces enabled by flexible scalp electronics and deep-learning algorithm
}

Musa Mahmood $^{1}$, Deogratias Mzurikwao ${ }^{2}$, Yun-Soung Kim ${ }^{1}$, Yongkuk Lee ${ }^{3}$, Saswat Mishra ${ }^{1}$, Robert Herbert ${ }^{1}$, Audrey Duarte ${ }^{4}$, Chee Siang Ang ${ }^{2}$ and Woon-Hong Yeo ${ }^{1,5,6, *}$

${ }^{1}$ George W. Woodruff School of Mechanical Engineering, Institute for Electronics and Nanotechnology, Georgia Institute of Technology, Atlanta, Georgia 30332, USA.

${ }^{2}$ School of Engineering and Digital Arts, University of Kent, Canterbury, Kent CT2 7NT, United Kingdom

${ }^{3}$ Department of Biomedical Engineering, Wichita State University, Wichita, Kansas 67260, USA

${ }^{4}$ School of Psychology, College of Sciences, Georgia Institute of Technology, Atlanta, Georgia 30332, USA

${ }^{5}$ Wallace H. Coulter Department of Biomedical Engineering, Parker H. Petit Institute for Bioengineering and Biosciences, Georgia Institute of Technology, Atlanta, Georgia 30332, USA.

${ }^{6}$ Neural Engineering Center, Center for Flexible and Wearable Electronics Advanced Research, Institute for Materials, Institute for Robotics and Intelligent Machines, Georgia Institute of Technology, Atlanta, Georgia 30332, USA.

*Corresponding author. Email: whyeo@gatech.edu (W.-H.Y.)

\begin{abstract}
Variation in human brains creates difficulty in implementing electroencephalography (EEG) into universal brain-machine interfaces (BMI). Conventional EEG systems typically suffer from motion artifacts, extensive preparation time, and bulky equipment, while existing EEG classification methods require training on a per-subject or per-session basis. Here, we introduce a fully portable, wireless, flexible scalp electronic system, incorporating a set of dry electrodes and flexible membrane circuit. Time domain analysis using convolutional neural networks allows for an accurate, real-time classification of steady-state visually evoked potentials on the occipital lobe. Simultaneous comparison of EEG signals with two commercial systems captures the improved performance of the flexible electronics with significant reduction of noise and electromagnetic interference. The two-channel scalp electronic system achieves a high information transfer rate $(122.1 \pm 3.53$ bits per minute) with six human subjects, allowing for a wireless, real-time, universal EEG classification for an electronic wheelchair, motorized vehicle, and keyboard-less presentation.
\end{abstract}




\section{Introduction}

Compared to other methods for electrophysiological monitoring of the brain, modern electroencephalography (EEG) systems provide non-invasive monitoring of brain electrical activity with fine temporal resolution, ease of use, and relatively low cost. Thus, EEG-based brainmachine interfaces (BMI) have been widely used for rehabilitation through control of prosthetic systems or substitutive control ${ }^{1-4}$. BMIs can restore freedom of movement and improve quality of life for chronic stroke survivors with locked-in syndrome (LIS) or persons with amyotrophic lateral sclerosis (ALS) or other severe motor disability ${ }^{5-8}$. The capture and classification of steady-state visually evoked potentials (SSVEP) is one potential strategy for therapeutic BMI ${ }^{9-12}$. For this type of interface, the subject is requested to focus on flickering stimuli (computer screens or external light sources), while an EEG system captures brain electrical activity from specific locations on the scalp ${ }^{13-15}$. These stimuli elicit frequency-dependent brain activity, and therefore arrays of stimuli may be used as an interface for subjects to gaze between for control over some target ${ }^{16-19}$. Conventional EEG setups for SSVEP acquisition use a hair-cap or crown-like setup, with many metal electrodes up to 256 channels $^{20-22}$. These systems are bulky and heavy, with uncomfortable, rigid metal scalp electrodes. These electrodes are often coupled with conductive gels or pastes to adequately capture the signal. Variance in scalp hair thickness and density cause variations in impedance between electrode locations ${ }^{23}$. Gel-based electrodes take considerable time to set up and require regular maintenance ${ }^{24,25}$. Additionally, water-based gels evaporate over time, causing decay in skin-electrode contact impedance ${ }^{15}$. Recent research in EEG design displays a trend toward wearable and wireless $\mathrm{EEG}^{26}$. These are preferable for day-to-day mobile EEG monitoring, with short setup times and excellent long-term performance provided adequate skin preparation with optimal amplifier, shielding, and electrode configurations ${ }^{25,27}$. New systems featuring dry electrodes perform as well as, if not better than, conventional systems, establishing strong groundwork toward a transition to dry electrode-based electrophysiological systems ${ }^{15,27,28}$. There is also an argument for the use of lightweight sensors and shorter leads in order to prevent dragging or movement artifacts that may occur with massy cap EEG systems ${ }^{29}$. However, the available mobile EEG systems ${ }^{30-33}$ are still quite bulky and use rigid electronic and structural components with a large number of electrodes, which are not comfortable for daily use and real-world applications. Here, we introduce the first example of a fully portable, wireless, flexible, skin-like hybrid scalp electronics (referred to as 'SKINTRONICS') that includes a low-profile, flexible circuit, an ultrathin aerosol-jet printed skin electrode, and three flexible conductive polymer electrodes for mounting on the hairy scalp (occipital lobe). The primary novelty is in the development of a fully integrated packaging of high-resolution EEG monitoring sensors and circuits within a miniaturized skin-conformal system. This soft electronic system is capable of improved EEG performance for idle, seated subjects due to its simplicity of design and compactness, isolated from motion artifacts caused by long wires and electrode movements, typically found from other EEG systems. SKINTRONICS offers maximal comfort and minimal setup time, requiring only two channels on the occipital lobe to measure SSVEP with competitive information transfer rates. We demonstrate a new capability to train deep convolutional neural networks $(\mathrm{CNN})$ offline and integrate them into wireless mobile devices for a real-time, universal EEG classification. Additionally, optimization of the system setup and classification model enables a highly competitive information transfer $\operatorname{rate}^{34}(122.1 \pm 3.53$ bits per minute) using only two channels, far fewer than most other systems achieving similar values. Applications for this system, including a powered wheelchair interface designed for subjects with LIS or other motor disability is demonstrated. With ever-improving mobile processing power, the combination of 
many-channel SKINTRONICS with integrated universal BMI, demonstrates possible implications for the use in portable EEG-based neuroprosthetics, real-time diagnosis of neurological disorders, or neuro-assisted learning (classroom support).

\section{Results \\ Device architecture and skin integration of a scalp electronic system}

Figure 1 shows an overview of the wireless, portable scalp electronics for SSVEP-based BMI. The photo in Fig. 1a captures the design concept of the flexible electronics that minimize the contact area (only 2 channels) on the scalp for a comfortable, dry EEG recording. The fully flexible, wearable system enables real-time long-range wireless data acquisition and accurate classification of SSVEP with a high information transfer rate from only two recording channels. Due to extreme mechanical compliance and small form factor, SKINTRONICS exhibits significant reduction of noise and electromagnetic interference, compared to the existing portable EEG systems with rigid electronic components ${ }^{33,35-37}$. Additionally, the use of conformal electronic components allows for easy wearability on the back of the neck or other bare skin locations. This soft system allows for a long-term wear versus other rigid devices with heavy plastic enclosures that have to be clipped-on or worn in other uncomfortable manner. Overall, the integrated electronics on the scalp, in conjunction with a deep-learning algorithm, demonstrates the feasibility for real-time, highly accurate in vivo BMI via SSVEP data from two channels. Fig. 1b shows the optimal electrode locations as determined by a deep CNN analysis of 32-channel EEG recording of SSVEP data (details in Methods; Fig. S1 and Section S1). The electrode positions in Fig. 1b $(\mathrm{O} 1, \mathrm{Oz}$, and $\mathrm{O} 2)$ were determined in preliminary tests to have consistently high SNR across all tested subjects. The deep CNN analysis is used to isolate the best electrodes from a larger cluster of electrodes, as it does not require prior knowledge of the signal type. This method is useful when the signal's features are difficult to decompose by conventional methods (e.g. power-spectrum analysis). The miniaturized, multi-channel flexible electronic system (Fig. 1c), encapsulated in a soft elastomeric membrane, was fabricated by using the combination of microfabrication techniques ${ }^{38}$, material transfer printing ${ }^{38}$, and hard-soft component integration ${ }^{39}$ (details in Methods; Fig. S2 and S3). This manufacturing process allows a skin-conformal, unobtrusive, and comfortable EEG device. The EEG recording setup for two channels $(\mathrm{O} 1-\mathrm{Oz}$ and $\mathrm{O} 2-\mathrm{Oz}$ ) incorporates an aerosol jet-printed skin-like electrode (Fig. 1d) and elastomeric hair electrodes (Fig. 1e). The highly conformal membrane electrode is placed at the right mastoid to serve as driven ground. We utilize a set of dry, flexible elastomeric electrodes (Cognionics) that make intimate contact to the hairy region, resulting in long-term EEG recording ${ }^{40}$. With adequate skin preparation, conformal contact provided by these electrodes allow for superior skin impedance (less than $20 \mathrm{k} \Omega$ ), and therefore lower noise in signal recording and transmission. In addition, there are only three scalp electrodes, effectively secured by using a single headband, which allows the electrode to splay its legs, separating and moving hair, to make effective contact with the scalp as demonstrated in Fig. 1e. A flow chart in Fig. 1f summarizes a high-level overview of data collection, processing, wireless transfer, and machine control (details of the EEG circuit in Fig. S4).

\section{Quantitative study of system mechanics and reliability}

To build a portable, wearable scalp electronics, intimate skin integration is critical while allowing the wearer to conduct everyday activity. The device must also maintain its ability to acquire highfidelity EEG along with wireless transmission of data while under bending. In this work, we 
subject the electronics to $180^{\circ}$ bending up to $1.3 \mathrm{~mm}$ in radius of curvature, well beyond the expected bending during skin deformation on the back of the neck. Similar mechanical tests are performed on the hair electrode and skin-like electrode to ensure long-term mechanical stability on the skin application. Computational finite element analysis (FEA) was used to design the electronic circuit and wearable electrode, which provided an optimal design to endure continuous mechanical deformation. Afterwards, a set of experiments demonstrated the mechanical reliability of the SKINTRONICS (Fig. 2), including microscope investigation, resistance measurement, and received signal strength indication (RSSI). Details of the computational and experimental study procedures appear in Methods. The flexible electronic circuit shows great mechanical stability upon cyclic bending along the vertical axis for the first location (Figs. 2a-d) and second location (Figs. 2e-h). Details of the bending points are summarized in Fig. S5a. With a complete folding of the device at the bending radius of $1.3 \mathrm{~mm}$ (Fig. 2a), the device shows no adverse effects, as supported by the FEA result (Fig. 2b) with negligible change in the maximum principal strain $(<$ $0.1 \%$ ). The change of electrical resistance of the interconnects in Fig. 2a is measured during the cyclic loading and unloading process (Fig. 2c), which also shows consistent resistance with the maximum change of $0.06 \Omega$. Additionally, the RSSI measurement monitors the wireless signal quality under $180^{\circ}$ bending up to 15 meters (Fig. 2d), showing that the device maintains connectivity at distances over 10 meters. Details of the mechanical bending configuration are shown in Fig. S5b. Another location of the electronics shows $180^{\circ}$ bending with the radius of 1.6 mm (Fig. 2e). The corresponding FEA in Fig. 2f shows similar results to the first location, and the interconnects are intact after bending. Fig. $\mathbf{2 g}$ shows the resistance measurements at this location where the maximum change in resistance is $0.09 \Omega$, indicating the stability of the interconnects during cyclic bending. The RSSI investigation (Fig. 2h) validates the device's functionality with distances up to 15 meters. The resistance measurements at each location after 100 bending cycles are shown in Fig. S5c, showing a slight increase in resistance, from 1.19 to $1.51 \Omega$ at location 1 , and from 1.08 to $1.38 \Omega$ at location 2. Furthermore, recorded SSVEP data (stimulus at $15.2 \mathrm{~Hz}$ ) compares the effect of cyclic bending of the electronics (Fig. 2i), which shows negligible change in the signal quality. The elastomeric hair-based electrodes underwent cyclic compression testing while monitoring resistance from the connector to a single leg of the electrode. The method is briefly described in Section S4, with the setup shown in Fig. S6. A maximum compression of 2 $\mathrm{mm}$, followed by release over a 6-second period (Fig. $\mathbf{2 j}$ ), was repeated for 1000 cycles, simulating the maximum compression exhibited during EEG recording. During a single cycle of compression, the peak change in resistance was only $4.1 \mathrm{k} \Omega$ (Fig. 2k). Even with 1,000 cycles, the uncompressed resistance changed a total of $4.46 \mathrm{k} \Omega$ (less than $10 \%$ of the initial resistance; Fig. S7). The conductive elastomeric electrodes showed excellent resilience to mechanical stress and were still capable of measuring EEG after the test was completed. These results are supported by a prior study that evaluated the robustness of these electrodes in a high-density multi-channel system ${ }^{41}$. For EEG recording, a skin-like silver electrode undergoes multi-modal bending and stretching on the skin (mastoid), considering the ultrathin epidermis on that area with time-dynamics movements with the ear. For bending tests, a setup like the one used in Fig. S5 for testing SKINTRONICS was used. Resistance change during bending was also measured, showing stability under complete folding at a small radius. The summary of the experimental and computational study in Figs. 2l$\mathbf{m}$ validates the structural safety of the printed electrode, with over $50 \%$ biaxial strain and up to $180^{\circ}$ bending at the radius of $250 \mu \mathrm{m}$ (details in Fig. S8 and S9). FEA results show the maximum strain under $1 \%$ in any region of the electrode, while microscopic observation captures no fracture lines. This evidence, in addition to our prior studies evidencing long-term reliability ${ }^{15}, 38,42$ 
regarding skin wearable electrodes demonstrate mechanical stability of SKINTRONICS device and electrodes for extended human use.

\section{Analysis of signal quality and classification accuracy}

The new electronic system, SKINTRONICS, is benchmarked against two state-of-the-art EEG monitoring devices. The first is a 32-channel EEG system (ActiveTwo System, Biosemi B.V.), using water-based conductive gels (SignaGel, Parker Laboratories) and the second is a wireless, portable system (BioRadio, Great Lakes NeuroTechnologies) that allows for 8 channels in a referential montage. We demonstrate the functionality and performance of the SKINTRONICS via the direct comparison with those commercial systems. The ActiveTwo 32-channel system uses $\mathrm{Ag} / \mathrm{AgCl}$ active electrodes to represent the gold standard, benchtop EEG system, while the BioRadio represents a wireless, handheld EEG and uses dry, flexible elastomeric electrodes (Cognionics) as a control to compare the performance of the SKINTRONICS. The detailed procedure for data acquisition is provided in Methods. Figure 3 summarizes the comparison of SSVEP captured using a single occipital lobe channel $(\mathrm{O} 1-\mathrm{Oz})$, by three different systems. The side-to-side comparison of $12.5 \mathrm{~Hz}$ SSVEP data captures the superior performance of the SKINRONICS (Figs. 3a-c) with the consistent and overlapping peaks of $12.5 \mathrm{~Hz}$ from the first channel $(\mathrm{O} 1-\mathrm{Oz})$. In these panels, periodograms from 50 consecutive 1-second sample windows with $128 \mathrm{~ms}$ overlap from a single subject are overlaid on a single graph to demonstrate the signal variance from each of the three devices. Signal-to-noise ratio (SNR) analysis (Figs. 3d-f) for all 6 subjects was performed offline in a numerical program (MATLAB, MathWorks). The method used for calculating SNR is provided. Among the three systems, the SKINTRONICS shows the highest average SNR $(46.6 \pm 2.16 \mathrm{~dB})$ from four SSVEP classes, measured with 6 human subjects. This shows that SKINTRONICS SNR is a significant improvement over the conventional gelbased system (ActiveTwo; $16.94 \pm 4.60 \mathrm{~dB}$ ) and the portable wireless system with dry electrodes (BioRadio; $28.89 \pm 2.28 \mathrm{~dB}$ ). Figures 3g-3k demonstrates each of four preprocessing and analysis methods with a 1.024-sec segment of $12.5 \mathrm{~Hz}$ SSVEP data. Two-channel (O1-Oz and O2-Oz) time-domain data (Fig. 3g) is converted to a single-window periodogram (Fig. 3h), PSDA (Fig. 3i), and CSDA (Fig. 3j). The details of PSDA and CSDA methods are shown in Section S6 and Section S7, respectively. The primary metric used to establish the efficacy of the EEG system is information transfer rate (ITR), calculated in bits per unit time, as shown in Methods. To further quantify the SKINTRONICS performance, we conducted a canonical correlation analysis (CCA; details in Section S5). Our system achieves an average accuracy of $89.6 \pm 2.1 \%$ at the shortest data length of 0.512 seconds for four SSVEP classes over 6 human subjects (original datasets in Fig. S10 and original accuracy of seven SSVEP classes in Fig. S11 with details in Methods). To improve upon the classification accuracy achieved using CCA, we utilized support vector machines (SVM) and $\mathrm{CNN}^{31}$. Feature extraction and classification techniques are detailed in Methods and Fig. S12. The summary of resulting accuracies from 4 datasets appears in Fig. 3k, which is based on 1-layer CNN analysis (architecture provided in Tables S2). These preliminary results suggest that the basic network most effectively extracts features from CSDA and timedomain data.

\section{Optimization of convolutional neural network}

One of the key study points of this work is to show the universal capability to classify SSVEP from two channels on any subject. Therefore, 6-fold cross-validation is performed on a subject-to-subject basis as described in Methods. The experimental classes, along with procedures for preparing training and test data are also provided. Here, convolutional neural networks are used to classify signals from the five classes. With shared weights, CNN can 
detect features on either channel at any location, while keeping the size of the network relatively small $^{43}$. From the most basic 1-layer CNN, a grid-search optimization method is used to optimize the model, as explained in Methods. As summarized in Fig. 4, multiple convolutional layers allow for detection of low-level features that cannot be detected with a single filter. It also allows for down-sampling and collection of relevant activations from previous layers, to be classified by a fully-connected layer. In Fig. 4a, we represent 'active' features using a gray-scale representation of the time-series data. Here, 50 2D-convolutions are performed across each channel, resulting in 100 outputs total. An additional 100 2D convolutions are performed on the second convolutional layer, resulting in the outputs shown in Fig. 4b. A fully connected layer of 1,024 units is produced, followed by a fully connected output layer with 5-class SoftMax activation (Fig. 4c). Mean accuracy results from SVM and CNN models for time-domain and CSDA data are shown in Fig. 4d and Fig. 4e, respectively. Of the four SVM kernel types tested, the highest accuracy results were chosen (cubic kernel) to be displayed for all results. This serves to establish universal applicability for the acquisition of SSVEP using only two-channel SKINTRONICS. The same subject-to-subject cross-validation scheme was used for all training models. The results show the SVM models able to achieve high accuracies with frequency-domain features for all kernel types. However, the linear SVM failed to classify time domain signals beyond trivial accuracies. Overall, the optimized 2-layer CNN was superior in all situations, due to its inherent ability to extract lowlevel features through shared weights, able to train to detect many features that SVM cannot. Based on this evidence, this system and classification model can be trained on a small group of individuals and used in universal SSVEP-based BMI applications. To further emphasize these offline results, a confusion matrix, representing the results from the shortest data window input (w $=0.512$ seconds) is summarized in Fig. 4f where the wireless scalp system achieves a high accuracy $(94.54 \pm 0.90 \%)$ for a corresponding ITR of $122.1 \pm 3.53$ bits per minute (Table 1). In comparison, real-time cue-guided SSVEP test data across all six subjects shows the accuracy of $94.01 \pm 3.6 \%$ (Fig. 4g). These results show a consistent level of accuracy even with real-time classification, which allows for precise control over different interface targets, including an electronic wheelchair, wireless mini-vehicle, and communication (presentation) tool.

\section{In vivo demonstration of wireless BMI with human subjects}

In this work, we demonstrate the feasibility of the SKINTRONICS for a portable, wireless BMI via SSVEP from 6 human subjects (Fig. 5). For the experiments, subjects were seated in front of the LED stimulus setup, about $0.8 \mathrm{~m}$ away from their head at eye level where all four stimuli are presented simultaneously (Fig. 5a). A subject conducts five tasks; gazing at each LED stimuli, and a null task (eyes closed, for alpha rhythms) and gazing four LED locations (Fig. 5b), while the SKINTRONICS securely conforms to the back of the subject's neck (Fig. 5c). The EEG data is recorded, displayed and saved in real-time, along with the corresponding classification outputs on an Android-based mobile device (Fig. 5d). A set of representative EEG data from 5 classes appears in Fig. 5e. A subject uses these data to control three target machines, including a wireless electronic wheelchair (Pronto, Invacare; Fig. 5f), a wireless mini-vehicle (Minidrone, Parrot; Fig. 5g), and a presentation software (PowerPoint 2016, Microsoft; Fig. 5h). Overall, subjects were able to achieve highly accurate and precise real-time control of three target machines with $94.01 \pm 3.6 \%$ accuracy at 0.512 -second intervals and $96.24 \pm 3.4 \%$ at 1.024 -second intervals. Subjects with various hair types and conditions were selected to ensure the electrode's performance in various scenarios (details of the BMI control scheme in Methods). Real-time, in vivo demonstration of the aforementioned machine controls with the SKINTRONICS are shown in Videos S1-S3. 


\section{Discussion}

The collection of materials presented here reports that a fully portable, wireless, ergonomic SKINTRONICS offers highly accurate, real-time monitoring of SSVEP on the scalp for a BMI. The flexible electronic system integrates a highly conformal wireless circuit on the back of the neck, a skin-like membrane electrode on the mastoid, and three flexible conductive electrodes on the occipital lobe. The extremely small form factor and associated portability of the low-power SKINTRONICS makes it less prone to interference and movement artifacts. The adherence to the skin and mechanically compliant electrodes allows for reasonable flexion and movements without any significant artifacts added to the EEG signals. Provided these advantages, locations of highest signal density may be targeted with fewer electrodes, producing high resolution signals from the areas of highest signal density. The electrode configuration is designed to target a consistently high SSVEP SNR across all subjects. A custom optimized algorithm using deep-learning CNN provides real-time, highly accurate classification of SSVEP and a highly efficient ITR with only two channels (122.1 \pm 3.53 bits per minute; Table 1), enabling precise control of a wireless wheelchair, motorized mini-car, and a presentation software. Even though other two systems ${ }^{30,44}$ show higher ITR than the SKINTRONICS, they used a full benchtop system with an EEG cap and 9-channel gel electrodes. In addition, unlike these systems, the SKINTRONICS only used flickering stimulus without any synchronization technique for enhanced accuracy, which requires additional work in the system. To mitigate a possible risk of collision or accident, the electronic wheelchair can use a risk prevention system that may incorporate infrared or other proximity sensors ${ }^{45}$. In addition, the wheelchair can be bound to a specific set of paths to prevent accidents arising from misclassification.

Overall, the collective result in this work is significant due to the decreased number of channels used when compared to other systems and is also fully integrated for comfort and a low profile. Due to the decreased number of channels, correlation-based analyses such as canonical correlation analysis (CCA) and task-related component analysis (TRCA) could not be used with 2 channels. This control demonstrated by healthy subjects may easily be learned by subjects with weak or nonexistent motor control (locked-in syndrome), requiring only movement of the eyes and closure of the eyelids. Using high-quality signals over a targeted two channels, combined with an optimum classification method, we can achieve a high accuracy with limited information, generalized enough to be used with any subject, demonstrating the possibility of a universal BMI. Additionally, the EEG may be reconfigured to monitor motor evoked potentials ${ }^{46}$ or motor imagination ${ }^{47}$ for motor-impaired subjects, which will be further studied as a future work on therapeutic applications. For these applications, different electrodes will be used that are optimized for the required electrode locations and target signal characteristics. Furthermore, more randomization in performance comparison between multiple systems will be conducted along with different control targets.

Collectively, this paper reports fundamental strategies to design an ergonomic, portable EEG system for a broad range of assistive devices, smart-home systems, and neuro-gaming interfaces. Future study would focus on investigation of fully elastomeric, wireless self-adhesive electrodes that can be mounted on the hairy scalp without any support from headgear, along with further miniaturization of the electronics to incorporate more electrodes for use with other studies.

\section{Methods}


Topographical mapping of SSVEP EEG data. The detailed methods for this process are recorded in Section S1 and Fig. S1 of the Supplementary Materials. The representative sample of a topographical scalp captures the largest signal weights on the $\mathrm{O} 1, \mathrm{O} 2$, and $\mathrm{Oz}$ channels (data of other EEG mapping from six human subjects appear in Fig. S1d). These results are consistent with other topographical maps in a prior work ${ }^{9}$. For a deep neural network analysis, data was sampled at $256 \mathrm{~Hz}$ and the reference was set at $\mathrm{Oz}$ for the best results. The optimal electrode location was determined by a CNN trained to classify from all 32-channels (details of architecture in Table S1). The trained weights for each channel could then be extracted and plotted using a topographical mapping algorithm provided by open EEGLAB (open source software) ${ }^{48}$. Details of this method are explained in Section S1. This multi-channel quantitative EEG study retrospectively verifies the optimal two electrode locations that were used in the two-channel EEG study.

Preparation of SKINTRONICS. The device preparation consisted of three stages. First, using microfabrication processes, the thin-film flexible circuit boards were constructed on a polydimethylsiloxane (PDMS)-coated four-inch silicon wafer and peeled-off for subsequent steps. ${ }^{49}$ The details of this process are given in Section S1. Second, the surface mount chip components were soldered onto exposed copper pads by reflow soldering using a solder paste (SMDLTLFP10T5, Chip Quik). Third, electrode integration and device encapsulation were conducted. For electrode integration, PDMS-insulated conductive film cables (HST-9805210, Elform) were used to connect the dry electrodes and the flexible device while using small amount of silver paint (Fast Drying Silver Paint, Ted Pella) as the adhesive for cable attachment. The assembled circuit was fully encapsulated with a low-modulus elastomer (Ecoflex 00-30, SmoothOn, Inc.) both to protect the circuit components and to provide the necessary adhesiveness and compliance for application on skin. Prior to the fabrication, the circuit design was validated on a printed board to confirm the optimized layout with matching antenna circuitry (Fig. S2a), along with the optimized components for a protocol of $2.45 \mathrm{GHz}$ Bluetooth low energy. The reflection coefficient $\left(\mathrm{S}_{11}\right)$, plotted in Fig. S2b, has a local minimum at $2.449 \mathrm{GHz}$ of $-29.68 \mathrm{~dB}$, which allows for excellent telemetry at reasonably large ranges. The details of the device fabrication and encapsulation processes appear in Section S2 and step-by-step illustration is shown in Fig. S3.

Recording of SSVEP. Flexible elastomeric electrodes (placed at O1, O2, and Oz) are connected to the SKINTRONICS through flexible thin film cables, which are routed under the headband and connected on the right side of the device. In addition, a skin-conformal, aerosol-jet printed electrode with improved skin adhesion reduces motion artifacts and enhances skin-electrode contact impedance. This electrode acts as a driven bias, therefore its ability to capture EEG is out of the scope of this paper and not tested. The SKINTRONICS wireless telemetry unit uses a Bluetooth low-energy microcontroller (nRF52832, Nordic Semiconductor) due to its high throughput, low latency, low power draw and wireless range ${ }^{39}$. The BLE protocol supports wireless synchronization of multiple devices to support greater numbers of channels ${ }^{50}$. Considering each SKINTRONICS can support up to 8 channels, more devices can be synchronized for measuring multi-EEG signals at different locations. For EEG recording, a front-end integrated circuit (ADS1299, Texas Instruments) was used, which is a low-noise 24-bit analog-to-digital converter with a built-in bias drive, while supporting up to eight differential-input channels (Fig. S4a). The design also incorporates second-generation low-noise instrumentation amplifiers as the first gain stage (INA828, Texas Instruments). A schematic and block diagram of the connections for the external amplifiers are shown in Fig. S4b, and the internal signal routing is listed in Fig. S4c. The optimal configuration for the first gain stage was set to $100 \mathrm{~V} / \mathrm{V}$ for use with dry electrodes, which provides an isolated low-noise, low-offset first gain stage. The internal 
programmable gain was set to 1 to minimize DC offset, harmonic distortion, and amplifier saturation. To improve common-mode rejection, the driven ground electrode's bias amplifier was configured as an open-loop. In this configuration, bias signals reached open-loop gain, which was the maximum possible gain achievable by the amplifier (Fig. S4d). Parasitic capacitive effects ${ }^{51}$ were limited through passive shielding with the elastomeric material on wiring and exposed electrode surfaces. A conventional bias electrode configuration is shown in Fig. S4e.

Computational mechanics. Finite element analysis was conducted using commercial software (Abaqus FEA, Dassault Systemes) to determine mechanical behavior under bending deformation at multiple locations. For the computational modeling, the following material properties $(E$ : Young's Modulus, $v$ : Poisson's Ratio) of $\mathrm{E}_{\mathrm{Cu}}=119 \mathrm{GPa}$ and $v_{\mathrm{Cu}}=0.34$ for copper; $\mathrm{E}_{\mathrm{PI}}=2.5 \mathrm{GPa}$ and $v_{\mathrm{PI}}=0.34$ for polyimide were used. ${ }^{52,53}$

Biaxial mechanical stretching. Biaxial stretching was used to test the stretchability of the skinlike flexible electrode used at the mastoid. Here, the electrode is placed in a custom-built biaxial stretcher (Fig. S8), where opposite ends of a completed electrode are silver-pasted to copper wires that are connected to a digital multi-meter for resistance measurements. Biaxial stretching caused the resistance to increase as expected as the interconnects thinned, followed by a fracture at over $60 \%$ strain (Fig. S9).

32-channel EEG recording. For determining precise electrode locations for placing a two channel EEG device on the scalp, a deep learning-based topographic mapping procedure is used. A 32channel EEG system (ActiveTwo System, Biosemi B.V., Amsterdam) was used to acquire data from subjects with active $\mathrm{Ag} / \mathrm{AgCl}$ electrodes interfaced with the scalp through conductive waterbased gel (Saline Base Signa Gel, Parker Laboratories, Inc., Fairfield, NJ). Impedances between measurement and ground electrodes were maintained below $10 \mathrm{k} \Omega$ during testing. The study involved 8 volunteers ages 18 to 40 and the study was conducted by following the approved IRB protocol (\# H17212) at Georgia Institute of Technology. Prior to the in vivo study, all subjects agreed with the study procedures and provided signed consent forms.

2-channel EEG recording. Target skin and scalp locations are cleaned with isopropyl alcohol before skin preparation. Abrasive skin preparation gel (NuPrep, Weaver and Co., United States) was gently applied to each of the electrode locations with a cotton swab to prepare the skin for electrode placement. Excess gel is removed using a gauze pad and the locations are cleaned with alcohol wipes before electrodes are applied. For the single skin electrode location, adhesive tape is used to remove dead skin cells from the surface and alcohol wipes are used to clean and prepare the skin. Impedances between the hair mount electrodes to ground were maintained below $20 \mathrm{k} \Omega$. Due to the use of flexible contacts of the scalp-mounted dry electrode and skin-like electrode on the mastoid, we maintained relatively low the skin-electrode contact impedance.

Calculation of signal-to-noise ratio (SNR). SNR was calculated from a continuously recorded 10 -second sample, using the computed periodogram with 2048-point $(8.192 \mathrm{sec})$ overlapping windows for each of the four stimuli. Data for each subject were recorded consecutively on the same day, starting with the ActiveTwo, followed by the BioRadio, and finally the SKINTRONICS. The recordings from all three devices were performed within two hours. The setup time for the ActiveTwo was about half an hour for each subject, while the BioRadio and SKINTRONICS were easier to set up due to the use of fewer electrodes (about 15 minutes to set up for each subject). Setup time includes skin preparation time and checking the electrode impedance before starting EEG recording. The time required to record all the training and test data was about 13.5 minutes, for each system, with each individual trial being 75 seconds, with a minute break in between each of the six trials. 
Calculation of information transfer rate (ITR). SSVEP BCI systems are generally assessed based on ITR, measured in bits/min and their classification accuracy. ${ }^{54}$ ITR is calculated as follows:

$$
I T R=\left(\log _{2} N+A \log _{2} A+(1-A) \log _{2}\left(\frac{1-A}{N-1}\right)\right) \times\left(\frac{60}{w}\right) \frac{\text { bits }}{\min }
$$

where $\mathrm{N}$ is the number of targets, $\mathrm{A}$ is the accuracy, and $w$ is the combined size of the classified windows in seconds, and the gaze shift time ${ }^{55}$. For each instruction relayed through the interface, the incoming EEG data was evaluated three times, every $64 \mathrm{~ms}$. Actions are taken if three consecutive windows are consistent, providing an additional layer of protection to prevent incorrect commands from being transmitted to targets. Using this method, the 4-class classification results using CCA results in an ITR of $77.63 \pm 5.32$ bits per minute, with window length, $\mathbf{w}=0.94$ $\mathrm{s}$, adjusted for gaze-shifting $(+300 \mathrm{~ms})$ and confusion protection $(+128 \mathrm{~ms})$. For testing classification performance, a total of 30 individual stimuli were presented, for a total of 0.704 seconds, which allowed for classification of three consecutive $0.512 \mathrm{~ms}$ samples. This was used as a safety mechanism for the control interface, where action would only be taken with a majority decision ( 2 or more classes match). Combined with gaze shift time, that brings the length of the window used up to 0.94 seconds $(0.512+0.3+0.128 \mathrm{~s})$. This same process was repeated for all the different window lengths. As a result, for each subject there were 450 samples of test data per trial for each window length, 90 for each class.

Feature extraction. Analysis includes comparison of time-domain and frequency-domain data using identical windows from the initial source in the form of single-window periodogram analysis and Welch power spectral density analysis (PSDA). A third, coherence-based method, crossspectral density analysis (CSDA) was also implemented due to its ability to analyze the coherence of multiple channels, allowing for more precise frequency decomposition versus single-channel PSDA. Time domain signals were preprocessed using $3^{\text {rd }}$ order Butterworth high-pass filter with a cutoff frequency of $4 \mathrm{~Hz}$ to remove DC offset and baseline drift. Due to the high quality of the source signals, no other digital filtration was found to significantly improve classification. These feature extraction methods are used to compare the learned feature-extraction capability of CNN versus manual feature extraction with SVM using various kernel functions (linear, quadratic, cubic, Gaussian).

Cross-validation of classification algorithms. For SVM and CNN classification, five subjects' training data was used to train the model and the remaining subject's data was used for evaluation. For the CNN, the training involved feeding a training batch of 256 and a test batch of 100 samples in each iteration. Following 3000 training iterations (approximately 57 epochs), or, if no validation improvement occurs after 150 training steps, the training terminates. The test sets are subsequently evaluated for accuracy.

Experimental classes. We measure SSVEP at four frequencies $(11.1,12.5,15.2$, and $16.7 \mathrm{~Hz}$ ), and alpha rhythms to test the robustness of a system to specifically decode SSVEP. We use alpha rhythms as the null class (no target interface action), intended for the user to relax their eyes in case of fatigue. Due to the overlapping frequency ranges between alpha rhythms $(8-12 \mathrm{~Hz})$ and intended SSVEP classes ${ }^{56}$, the decoding process becomes more complex and error-prone to conventional machine learning techniques. Therefore, SSVEP frequencies below $11.1 \mathrm{~Hz}$ were not used due to frequency overlap with alpha rhythms. It was studied that the risk of short bursts of alpha rhythms could cause false positive results in these ranges ${ }^{57}$. The converse case is also a concern, where intentional alpha rhythms (subject has eyes closed) are incorrectly interpreted as SSVEP within the alpha frequency band. Despite many prior works showing SSVEP-based 
systems within the alpha rhythm range, there has been limited study regarding confusion between SSVEP and alpha rhythm classification. Frequencies above $16.7 \mathrm{~Hz}$ were considered (18.5 and 20 $\mathrm{Hz}$ ) in a 7-class SSVEP system and the average classification accuracy across all subjects was summarized in Fig. S10. However, these frequencies were not included in the final system as they were considered extraneous, and some subjects had trouble focusing on these higher frequency stimuli. The stimulus for this application included four green LEDs arranged on a 3D-printed hollow ring fixture. A microcontroller (nRF51, Nordic Semiconductor, Oslo, Norway) controls the timings of the LEDs using a real-time operating system (FreeRTOS, open-source software) to ensure correct timings. The timings to toggle each LED were set as $45 \mathrm{~ms}, 40 \mathrm{~ms}, 33 \mathrm{~ms}$, and 30 ms, corresponding to $11.1 \mathrm{~Hz}, 12.5 \mathrm{~Hz}, 15.2 \mathrm{~Hz}$, and $16.7 \mathrm{~Hz}$ respectively.

Preparation of training and test data. For all experiments, a $250 \mathrm{~Hz}$ sampling rate was used with SKINTRONICS. The procedure for gathering training data involved a continuous EEG recording with 15 seconds for each class, separated by auditory cues. The classes were recorded in order of alpha rhythms, for the first 15 seconds, followed by 15 seconds of gazing at four different LED stimuli. Here, each recording where all five classes are performed constitutes a single trial. This trial was performed 6 times for each subject. Note that the experimental method for gathering training data is not the same as the experimental testing method. Empirical data from the SKINTRONICS suggested that training from a continuous stimulus was significantly more effective use of training data than a faster $(<1$ second) cue-guided task. This may be because the relevant SSVEP features required for training are most optimal during these longer sessions of looking at the stimulus. To train the SVM and CNN models, the training data were sub-divided into window sizes of $128,192,256,384,512$ data points (corresponding to $0.512,0.768,1.024$, $1.536,2.048 \mathrm{sec}$ data windows) to gauge changes in accuracy over different window sizes. The recording procedure for training and test data allowed for an additional 0.3 seconds for gazeshifting between stimuli. From the training set, there were 2700 samples from each subject used, 450 samples from each of the 6 recordings. Therefore, for 5 subjects, a single training epoch consisted of training on 13,500 samples.

Grid search optimization of CNN. In all CNN models, the filters and other trained variables were optimized using an optimization algorithm with a constant learning rate (Adam, learning rate $\left.=0.001, \beta_{1}=0.9, \beta_{2}=0.999\right)^{58}$, and error was calculated using the cross-entropy loss function. The input to the network is a $2 \mathrm{D}$ array of $(\mathrm{n}, 2)$, where $\mathrm{n}$ is the number of data points for a window sampled at $250 \mathrm{~Hz}$ (e.g. $\mathrm{n}=128$, for a window length of $0.512 \mathrm{~s}$ ), and the 2 represents each of the two channels. This work optimizes the baseline CNN (architecture in Table S2) through two stages of improvements, adjusting components and hyperparameters on a single layer. A grid search approach was used because training for such a small dataset could be completed in a few minutes on consumer hardware, and it was reasonably efficient to find an optimal solution. First the optimum parameters are determined for a CNN with 1 convolutional layer and 1 fully connected layer before the output layer. This model is then expanded to multiple layers, as needed, to further improve accuracy. Details of optimization procedure via grid search appear in Section S8 and illustrated in Fig. S13-S16 and Table S3-S8 in Supplementary Materials. The optimized CNN for time-domain (2-CNN-TD) and CSDA (2-CNN-CSDA) data can be found in Table S9 and Table S10 respectively. The optimization of CNN results in enhanced classification accuracy from 6 subjects (Table S11-S14). To demonstrate the device performance, the same optimized CNN procedure was performed on data from two commercial systems (BioRadio and ActiveTwo) and summarized in Table S15-S16 and Fig. S17. 
BMI control scheme. An Android-based operating system was selected to demonstrate functionality and feasibility of a fully portable, wireless BMI. The SSVEP data transmitted from the SKINTRONICS with short electrode cables (Table S17) was received by a Bluetooth enabled smartphone (Samsung Galaxy S7). The data was preprocessed and fed into the trained CNN for classification on the smartphone. The output of the classification was used to wirelessly control the interface target. For the electronic wheelchair and wireless vehicle, the methods of control are the same as they are both two-wheel drive vehicles. When the subject has their eyes closed, the vehicle does not move, and only begin to move once SSVEP is classified by the central processing device. The top LED $(11.1 \mathrm{~Hz})$ is used to program the forward motion of the wheelchair and vehicle. The right-facing $15.2 \mathrm{~Hz}$ LED results in a counter-clockwise rotation, while the left-facing 12.5 Hz results in a clockwise rotation. The final $16.7 \mathrm{~Hz}$ LED operates the reverse functionality. For the presentation control interface, the top LED initiates the start of the presentation, the right LED proceeds forward through the slides, and the left LED reverts to the previous slide. Lastly, the bottom LED terminates the presentation. Note that six subjects participated in the BMI control study (their hair information and associated EEG SNR in Table S18). Overall, this BMI control demonstration with the wireless, portable SKINTRONICS shows the potential for other neurointerface applications, as the controls can be easily reassigned to any target with equivalent or fewer targets. Additionally, this method can be scaled up to include a greater number of classes, which was previously not possible due to a lack of signal quality.

\section{Data Availability}

All data supporting the findings of this study are available upon reasonable request from the corresponding author.

\section{Code Availability}

All code used to generate the results in this paper is available upon reasonable request from the corresponding author.

\section{Acknowledgments}

W.-H.Y. acknowledges a grant by the Fundamental Research Program (Project PNK5061) of Korea Institute of Materials Science, a grant from the Samsung Global Technology Center, and support from the Institute for Electronics and Nanotechnology, a member of the National Nanotechnology Coordinated Infrastructure, which is supported by the National Science Foundation (Grant ECCS-1542174).

\section{Author contributions}

M.M. and W.-H.Y. designed the research project; M.M., D.M., Y.K., Y.L., R.H., A.S., S.M., C.S.A., and W.-H.Y. performed research; M.M., D.M., A.D., C.S.A., and W.-H.Y. analyzed data; and M.M., D.M., C.S.A., and W.-H.Y. wrote the paper.

\section{Competing interests}

W.-H.Y. and M.M. are the inventors on a pending US patent application. 


\section{References}

1. Garcia, J.O., Srinivasan, R. \& Serences, J.T. Near-real-time feature-selective modulations in human cortex. Curr Biol 23, 515-522 (2013).

2. Zhu, D., Bieger, J., Garcia Molina, G. \& Aarts, R.M. A survey of stimulation methods used in SSVEP-based BCIs. Comput Intell Neurosci, 702357 (2010).

3. Luo, A. \& Sullivan, T.J. A user-friendly SSVEP-based brain-computer interface using a time-domain classifier. J Neural Eng 7, 26010 (2010).

4. Stamps, K. \& Hamam, Y. Towards Inexpensive BCI Control for Wheelchair Navigation in the Enabled Environment - A Hardware Survey. Lect Notes Artif Int 6334, 336-345 (2010).

5. Guger, C. et al. How Many People Could Use an SSVEP BCI? Front Neurosci 6, 169 (2012).

6. Ramos-Murguialday, A. et al. Brain-machine interface in chronic stroke rehabilitation: a controlled study. Ann Neurol 74, 100-108 (2013).

7. Soekadar, S.R., Birbaumer, N., Slutzky, M.W. \& Cohen, L.G. Brain-machine interfaces in neurorehabilitation of stroke. Neurobiol Dis 83, 172-179 (2015).

8. Nicolas-Alonso, L.F. \& Gomez-Gil, J. Brain computer interfaces, a review. Sensors (Basel) 12, 1211-1279 (2012).

9. Bin, G., Lin, Z., Gao, X., Hong, B. \& Gao, S. The SSVEP topographic scalp maps by canonical correlation analysis. Conf Proc IEEE Eng Med Biol Soc 2008, 3759-3762 (2008).

10. Wang, Y., Wang, R., Gao, X., Hong, B. \& Gao, S. A practical VEP-based brain-computer interface. IEEE Trans Neural Syst Rehabil Eng 14, 234-239 (2006).

11. Wang, Y., Gao, X., Hong, B., Jia, C. \& Gao, S. Brain-computer interfaces based on visual evoked potentials. IEEE Eng Med Biol Mag 27, 64-71 (2008).

12. Kelly, S.P., Lalor, E.C., Reilly, R.B. \& Foxe, J.J. Visual spatial attention tracking using high-density SSVEP data for independent brain-computer communication. IEEE Trans Neural Syst Rehabil Eng 13, 172-178 (2005).

13. Herrmann, C.S. Human EEG responses to $1-100 \mathrm{~Hz}$ flicker: resonance phenomena in visual cortex and their potential correlation to cognitive phenomena. Experimental Brain Research 137, 346-353 (2001).

14. Wang, Y., Wang, Y.T. \& Jung, T.P. Visual stimulus design for high-rate SSVEP BCI. Electronics Letters 46 (2010).

15. Norton, J.J.S. et al. Soft, curved electrode systems capable of integration on the auricle as a persistent brain-computer interface. Proceedings of the National Academy of Sciences 112, 3920-3925 (2015).

16. Beverina, F., Palmas, G., Silvoni, S., Piccione, F. \& Giove, S. User adaptive BCIs: SSVEP and P300 based interfaces. PsychNology Journal 1 (2003).

17. Kronegg, J., Voloshynovskiy, S. \& Pun, T. Analysis of bit-rate definitions for BrainComputer Interfaces. HCI '05: Proceedings of the 2005 International Conference on Human-Computer Interaction, 40-46 (2005).

18. Lin, Z., Zhang, C., Wu, W. \& Gao, X. Frequency recognition based on canonical correlation analysis for SSVEP-based BCIs. IEEE Trans Biomed Eng 54, 1172-1176 (2007).

19. Martinez, P., Bakardjian, H. \& Cichocki, A. Fully OnlineMulticommand Brain-Computer Interface with Visual Neurofeedback Using SSVEP Paradigm. Comput Intell Neurosci, 94561 (2007). 
20. Bin, G., Gao, X., Yan, Z., Hong, B. \& Gao, S. An online multi-channel SSVEP-based brain-computer interface using a canonical correlation analysis method. J Neural Eng 6, 046002 (2009).

21. Wang, Y.T., Wang, Y., Cheng, C.K. \& Jung, T.P. Measuring steady-state visual evoked potentials from non-hair-bearing areas. Conf Proc IEEE Eng Med Biol Soc 2012, 18061809 (2012).

22. Cecotti, H. A time-frequency convolutional neural network for the offline classification of steady-state visual evoked potential responses. Pattern Recognition Letters 32, 1145-1153 (2011).

23. McAdams, E.T., Jossinet, J., Lackermeier, A. \& Risacher, F. Factors affecting electrodegel-skin interface impedance in electrical impedance tomography. Medical \& Biological Engineering \& Computing 34, 397-408 (1996).

24. Searle, A. \& Kirkup, L. A direct comparison of wet, dry and insulating bioelectric recording electrodes. Physiol Meas 21, 271-283 (2000).

25. Li, G., Wang, S. \& Duan, Y.Y. Towards gel-free electrodes: A systematic study of electrode-skin impedance. Sensors and Actuators B: Chemical 241, 1244-1255 (2017).

26. Lin, C.T. et al. Review of wireless and wearable electroencephalogram systems and braincomputer interfaces--a mini-review. Gerontology 56, 112-119 (2010).

27. Salvo, P. et al. A 3D printed dry electrode for ECG/EEG recording. Sensors and Actuators A: Physical 174, 96-102 (2012).

28. Stauffer, F. et al. Skin Conformal Polymer Electrodes for Clinical ECG and EEG Recordings. Adv Healthc Mater 7, e1700994 (2018).

29. Tallgren, P., Vanhatalo, S., Kaila, K. \& Voipio, J. Evaluation of commercially available electrodes and gels for recording of slow EEG potentials. Clin Neurophysiol 116, 799-806 (2005).

30. Nakanishi, M. et al. Enhancing Detection of SSVEPs for a High-Speed Brain Speller Using Task-Related Component Analysis. IEEE Trans Biomed Eng 65, 104-112 (2018).

31. Kwak, N.S., Muller, K.R. \& Lee, S.W. A convolutional neural network for steady state visual evoked potential classification under ambulatory environment. PLoS One 12, e0172578 (2017).

32. y Alvarez, G.D. et al. Wireless EEG System Achieving High Throughput and Reduced Energy Consumption Through Lossless and Near-Lossless Compression. IEEE transactions on biomedical circuits and systems 12, 231-241 (2018).

33. Lin, C.-T., Chiu, C.-Y., Singh, A.K., King, J.-T. \& Wang, Y.-K. A Wireless Multifunctional SSVEP-Based Brain Computer Interface Assistive System. IEEE Transactions on Cognitive and Developmental Systems (2018).

34. Schlogl, A., Keinrath, C., Scherer, R. \& Furtscheller, P. in Neural Engineering, 2003. Conference Proceedings. First International IEEE EMBS Conference on 641-644 (IEEE, 2003).

35. Xu, J. \& Zhong, B. Review on portable EEG technology in educational research. Computers in Human Behavior 81, 340-349 (2018).

36. Shi, M. et al. in Advanced Computational Intelligence (ICACI), 2018 Tenth International Conference on 249-253 (IEEE, 2018).

37. Chen, X., Zhao, B., Wang, Y., Xu, S. \& Gao, X. Control of a 7-DOF Robotic Arm System With an SSVEP-Based BCI. International journal of neural systems, 1850018 (2018). 
38. Mishra, S. et al. Soft, conformal bioelectronics for a wireless human-wheelchair interface. Biosensors and Bioelectronics 91, 796-803 (2017).

39. Lee, Y. et al. Wireless, intraoral hybrid electronics for real-time quantification of sodium intake toward hypertension management. Proc Natl Acad Sci 115, 5377-5382 (2018).

40. Chi, Y.M. et al. in International Conference on Augmented Cognition 649-655 (Springer, 2013).

41. Mullen, T.R. et al. Real-time neuroimaging and cognitive monitoring using wearable dry EEG. IEEE Transactions on Biomedical Engineering 62, 2553-2567 (2015).

42. Lee, Y. et al. Soft electronics enabled ergonomic human-computer interaction for swallowing training. 7, 46697 (2017).

43. Krizhevsky, A., Sutskever, I. \& Hinton, G.E. Imagenet classification with deep convolutional neural networks. Advances in Neural Information Processing Systems (2012).

44. Chen, X. et al. High-speed spelling with a noninvasive brain-computer interface. Proc Natl Acad Sci U S A 112, E6058-6067 (2015).

45. Ji, Y., Hwang, J. \& Kim, E.Y. An intelligent wheelchair using situation awareness and obstacle detection. Procedia-social and behavioral sciences 97, 620-628 (2013).

46. Ma, T. et al. The hybrid BCI system for movement control by combining motor imagery and moving onset visual evoked potential. Journal of neural engineering 14, 026015 (2017).

47. Pfurtscheller, G. \& Neuper, C. Motor imagery and direct brain-computer communication. Proceedings of the IEEE 89, 1123-1134 (2001).

48. Delorme, A. \& Makeig, S. EEGLAB: an open source toolbox for analysis of single-trial EEG dynamics including independent component analysis. J Neurosci Methods 134, 9-21 (2004).

49. Kim, Y.S. et al. Scalable Manufacturing of Solderable and Stretchable Physiologic Sensing Systems. Adv Mater 29 (2017).

50. Bideaux, A., Zimmermann, B., Hey, S. \& Stork, W. Synchronization in wireless biomedical-sensor networks with Bluetooth Low Energy. Current Directions in Biomedical Engineering 1, 73-76 (2015).

51. Mastinu, E., Ortiz-Catalan, M. \& Hakansson, B. Analog Front-Ends comparison in the way of a portable, low-power and low-cost EMG controller based on pattern recognition EMBC 2015. Conf Proc IEEE Eng Med Biol Soc 2015, 2111-2114 (2015).

52. Zhang, Y., Zhou, G., Jin, J., Wang, X. \& Cichocki, A. Frequency recognition in SSVEPbased BCI using multiset canonical correlation analysis. Int J Neural Syst 24, 1450013 (2014).

53. Xu, R. et al. Fabric-based stretchable electronics with mechanically optimized designs and prestrained composite substrates. Extreme Mechanics Letters 1, 120-126 (2014).

54. Obermaier, B., Neuper, C., Guger, C. \& Pfurtscheller, G. Information transfer rate in a five-classes brain-computer interface. IEEE Trans Neural Syst Rehabil Eng 9, 283-288 (2001).

55. Shannon, C.E. \& Weaver, W. The mathematical theory of information. (1949).

56. Goldman, R.I., Stern, J.M., Engel, J., Jr. \& Cohen, M.S. Simultaneous EEG and fMRI of the alpha rhythm. Neuroreport 13, 2487-2492 (2002).

57. Wang, Y., Wang, R., Gao, X. \& Gao, S. in Proceedings. 2005 First International Conference on Neural Interface and Control, 2005. 37-39 (2005). 
58. Kingma, D.P. \& Ba, J. in ArXiv e-prints (2014).

59. Bevilacqua, V. et al. A novel BCI-SSVEP based approach for control of walking in Virtual Environment using a Convolutional Neural Network. Ieee Ijcnn, 4121-4128 (2014).

60. Volosyak, I. SSVEP-based Bremen-BCI interface--boosting information transfer rates. $J$ Neural Eng 8, 036020 (2011). 

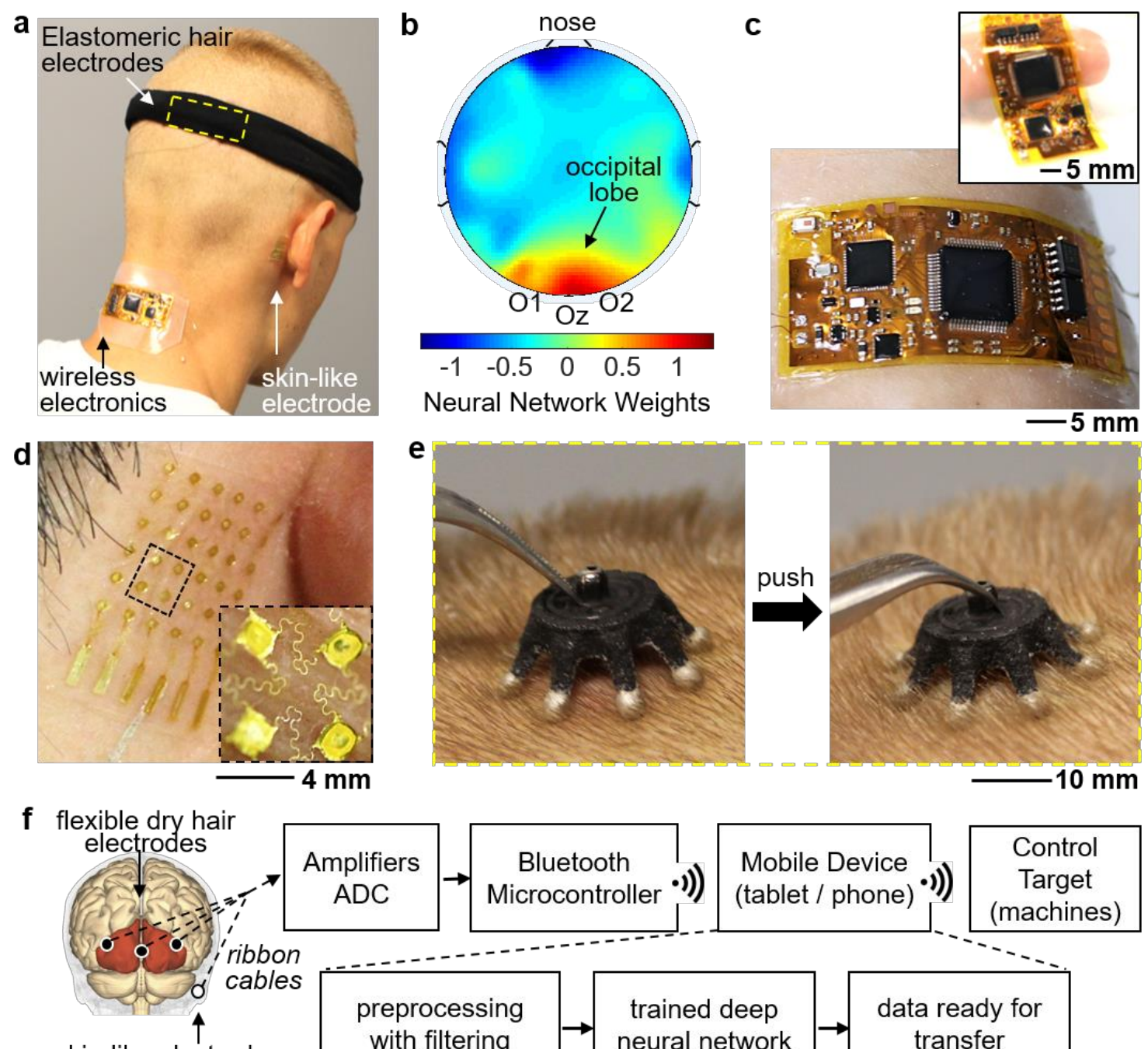

skin-like electrode

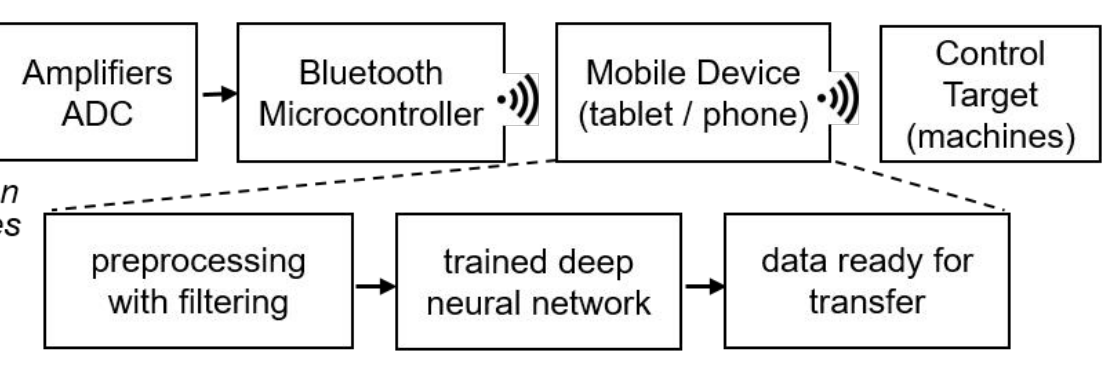

Figure 1. Overview of the system architecture featuring fully portable and wireless scalp electronics. a, Photo of a subject who has flexible wireless electronics (SKINTRONICS) conformed to the back of the neck with dry hair electrodes under a fabric headband and a membrane electrode on the mastoid, connected via thin film cables. b, Neural network-based topographical map that indicates EEG signal amplitudes. c, Photo capturing the ultrathin, flexible wireless electronics on skin, with an inset demonstrating device flexibility while handling. d, Aerosol-jet printed stretchable, skin-like electrode with an open-mesh structure (inset). e, Images showing gentle splaying of the conductive flexible elastomer legs of a dry hair electrode when slight downward pressure is applied, allowing the legs to separate the hair and the $\mathrm{Ag} / \mathrm{AgCl}$ tipped legs to achieve good contact with the scalp. f, Illustration (left) showing electrode positions on a posterior view of the brain and highlighted occipital lobe and flow chart (right) describing the entire process of EEG-enabled brain-machine interfaces. 

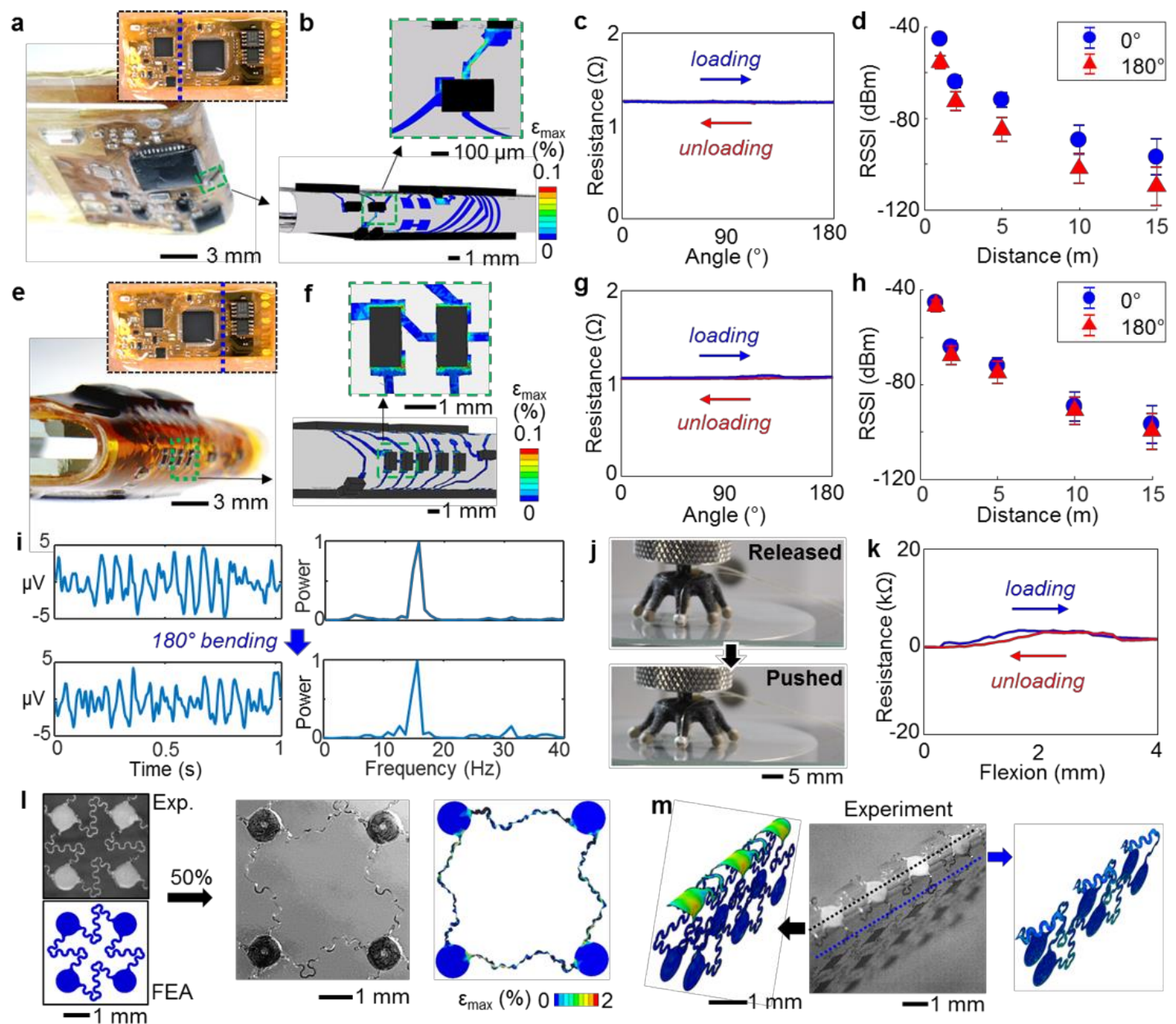

Figure 2. Mechanical flexibility and stretchability of the scalp electronics. a, Photo of the electronics bent at first location along the vertical axis, as shown in the inset photo, demonstrating $180^{\circ}$ bending (radius of curvature: $1.3 \mathrm{~mm}$ ). $\mathrm{b}$, FEA of fine mesh structure simulating $180^{\circ}$ bending with same radius resulting in minimal strain at the interconnects (scale bar: maximum principal strain). c, Measurement of electrical resistance for the device under cyclic bending between 0 and $180^{\circ}$, showing negligible changes in resistance. d, RSSI response at $0^{\circ}$ and $180^{\circ}$ with different distances up to 15 meters ( $n=3$ samples). e, Device bent at the second location along the vertical axis, as shown in the inset photo, between ADC and amplifiers (radius of curvature: $1.3 \mathrm{~mm}$ ). $\mathrm{f}$, FEA of the flexible structure simulating $180^{\circ}$ bending with minimal strain (scale bar: maximum principal strain). g, Measurement of cyclic bending effect on the device in e, by recording the change of electrical resistance. h, RSSI response up to 15 meters at $0^{\circ}$ and $180^{\circ}$ bending. i, 15.2 $\mathrm{Hz}$ SSVEP data recorded without (top) and with $180^{\circ}$ bending (bottom). j, Hair-based elastomer electrode under compression. $\mathrm{k}$, Corresponding load and unload curves showing a negligible peak change in resistance over a 6 -second cycle. 1 , Subunit of a skin-mounted electrode under $100 \%$ stretching in experiment and FEA, showing no mechanical failure. $\mathrm{m}, 180^{\circ}$ bending test of the electrode, showing less than $2 \%$ strain in FEA and no mechanical defects in experimental observation. 

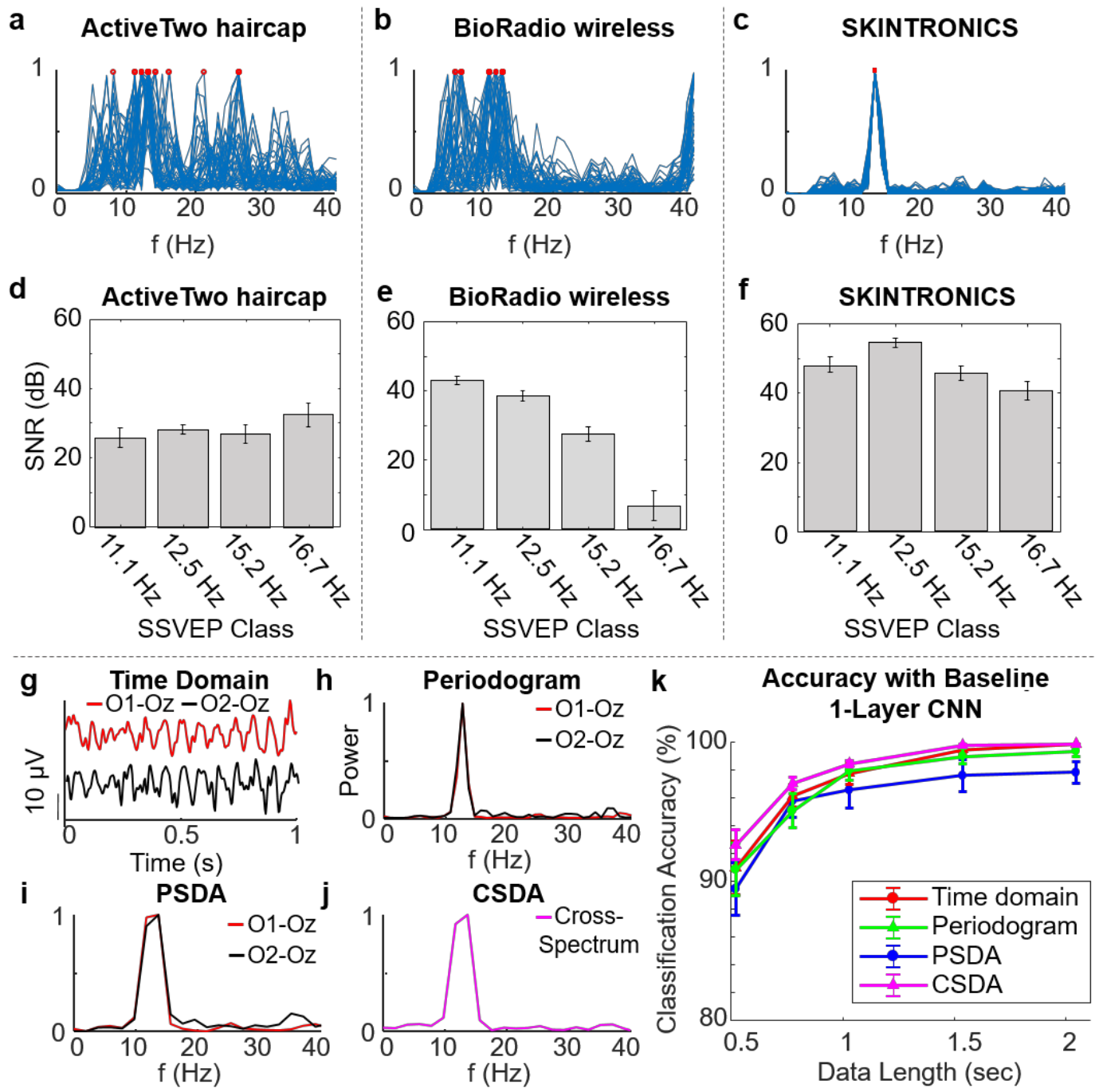

Figure 3. Comparison of device signal quality and classification accuracy. Three data sets for a conventional EEG system (ActiveTwo; $1^{\text {st }}$ column), bulky clip-on wireless system (BioRadio; $2^{\text {nd }}$ column), and our 2-channel SKINTRONICS ( ${ }^{\text {rd }}$ column). a-c, Periodograms of 50 samples from $12.5 \mathrm{~Hz}$ SSVEP signals overlaid over each other, with peaks highlighted with red circles; this comparison illustrates our device's ability to precisely target and capture SSVEP signals without excessive noise. d-f, Average signal-to-noise ratio (SNR) with four SSVEP classes, across all six tested subjects. Error bars represent standard error of the mean ( $\mathrm{n}=60$ recordings; 6 subjects, 10 recordings each). g, Two-channel SSVEP data at $12.5 \mathrm{~Hz}$ in time domain. h, Further preprocessing in the form of frequency-analysis including a single-window periodogram. i, Welch power-spectral density analysis (PSDA) overlapping periodogram. $\mathrm{j}$, Cross-spectral density analysis (CSDA) of both channels. Note that the cross-spectral input includes the normal Welch power spectrum, resulting in three features. $\mathrm{k}$, Comparison of classification accuracy between four preprocessing methods, shown in (g-j). The points on the graph are the mean accuracies from 36 trials from all 6 test subjects. Error bars represent standard error of the mean $(n=36$ trials). 


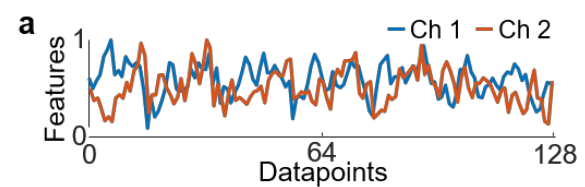

b
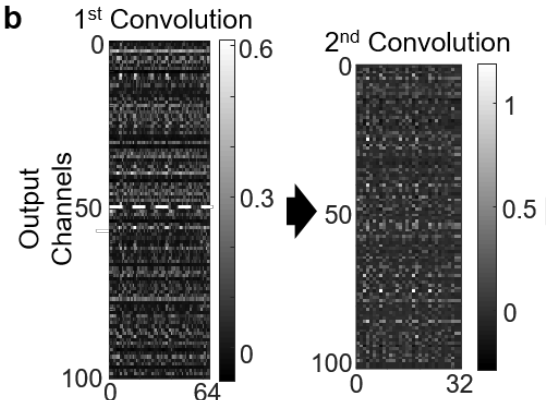

d

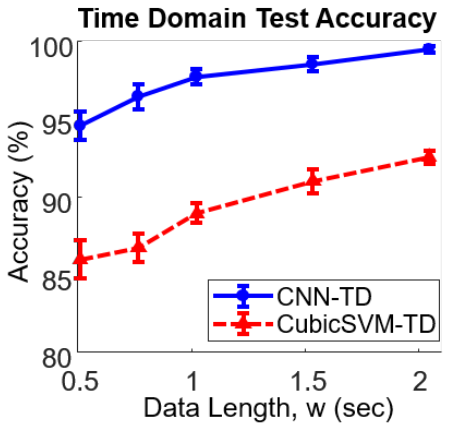

f

Offline Test, $\mathrm{N}=\mathbf{4 5 0}$ $(94.54 \pm 0.9 \%, w=0.512+0.492 \mathrm{~s})$

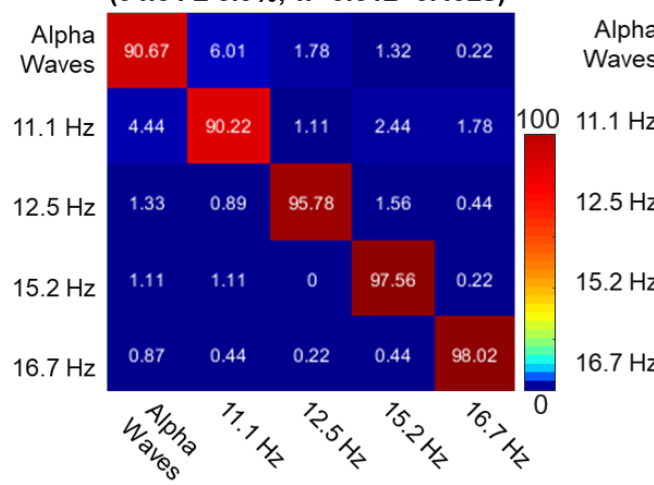

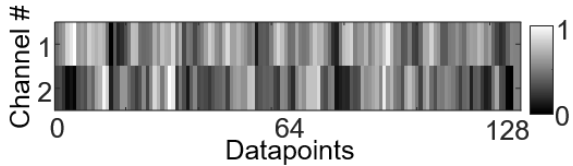

C Softmax Output:

Class Prob

a-waves $\quad 0.0000$

$11.1 \mathrm{~Hz} \quad 0.0000$

$12.5 \mathrm{~Hz} \quad 0.9989$

$15.2 \mathrm{~Hz} \quad 0.0000$

$16.7 \mathrm{~Hz} \quad 0.0011$

e

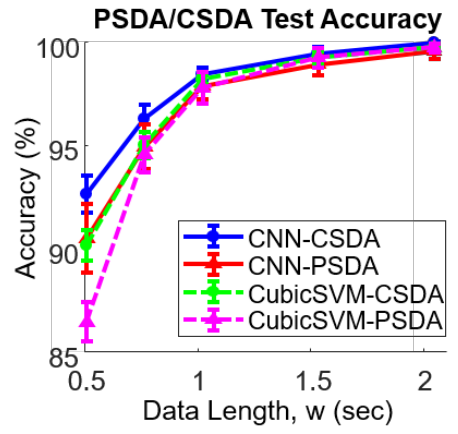

g

Real-Time Test, $\mathbf{N}=150$

$(94.01 \pm 3.6 \%, w=0.512+0.492 \mathrm{~s})$

$\begin{array}{lllll}91.99 & 4.13 & 2.18 & 0.73 & 0.97\end{array}$

\begin{tabular}{l|l|lll}
\hline 5.44 & 90.71 & 1.36 & 1.36 & 1.13
\end{tabular}

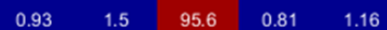

$\begin{array}{lllll}0.91 & 0.57 & 0.68 & 96.59 & 1.25\end{array}$

$\begin{array}{lllll}0.73 & 1.09 & 0.73 & 1.82 & 95.63\end{array}$

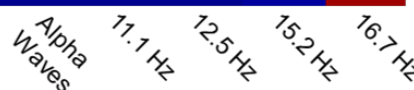

Figure 4. EEG classification with CNN. a, High-pass filtered raw EEG data in time domain with features linearly rescaled between 0 and 1, labeled as SSVEP class ' $12.5 \mathrm{~Hz}$ ', along with a representative greyscale image. $b$, Greyscale representation of output weights in the two layers of CNN as well as in the fully connected layer using the 2-CNN model for time domain data. c, Table of softmax class outputs, indicating a correct choice with $99.89 \%$ probability. d, CNN and SVM classification test accuracy using time domain data and cross-validating across all subjects. e, CNN and SVM test accuracy on frequency-domain data, cross-validating across all subjects, with window lengths from 0.512 to 2.048 seconds. The displayed results are from the strongest SVM of the four tested (cubic kernel). f, Confusion matrix representing results from offline accuracy test of time-domain data of data window length $w=0.512 \mathrm{~s}$, with an overall accuracy of $94.54 \%$ ( $\mathrm{n}=2700$ samples; 6 subjects, 450 samples each). g, Confusion matrix representing results from real-time accuracy test of time-domain data of length $w=0.512 \mathrm{~s}$, with an overall accuracy of 94.01\% ( $\mathrm{n}=2700$ samples; 6 subjects, 450 samples each). 

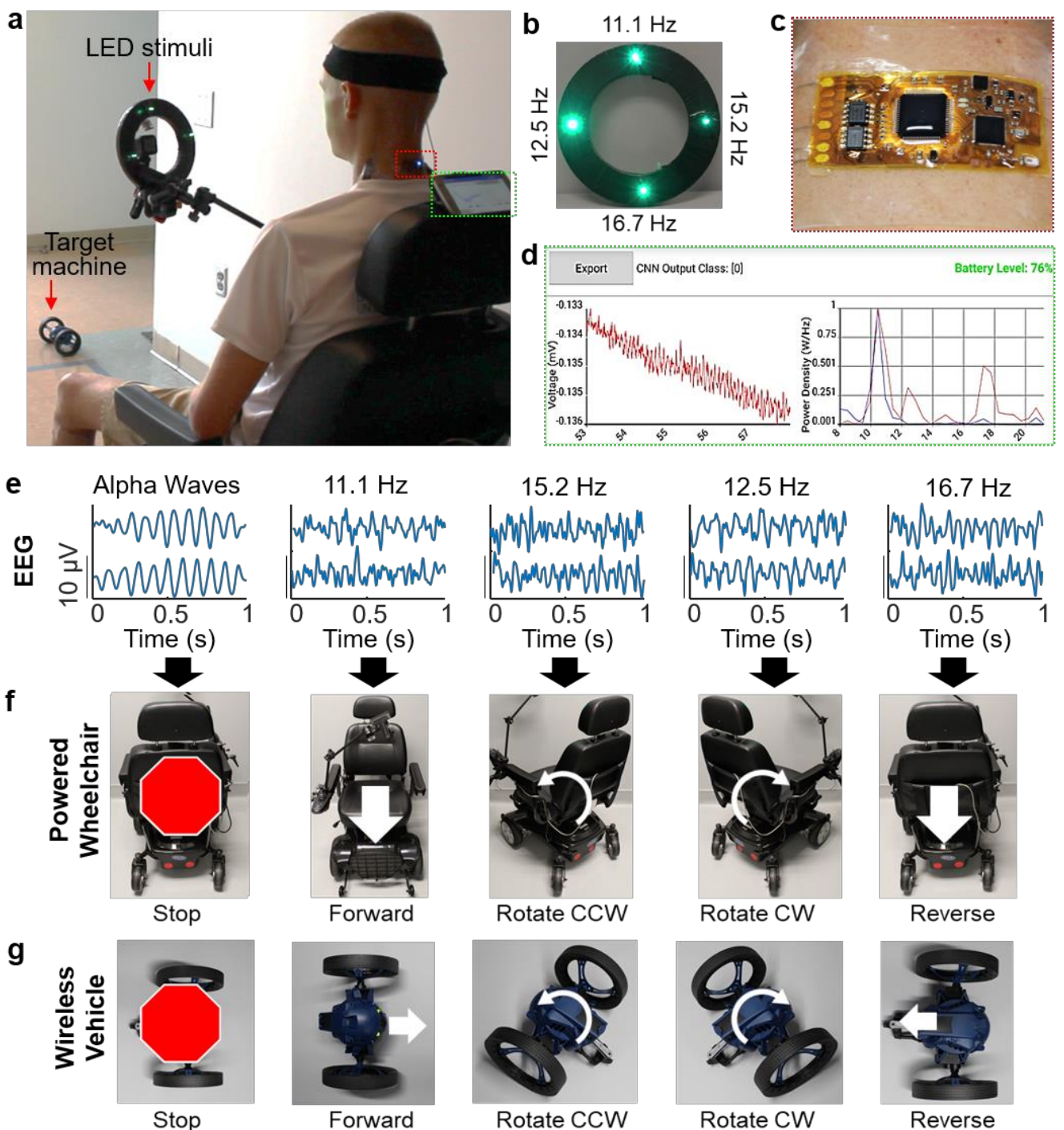

Rotate CW

h
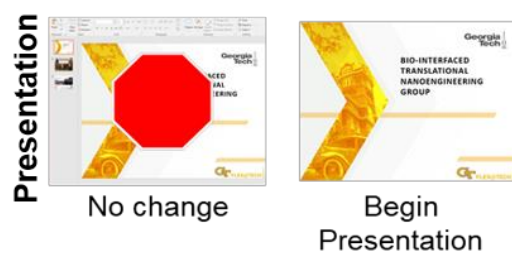

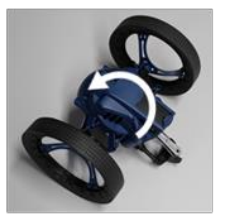

Rotate CCW

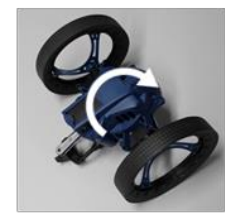

Rotate CW

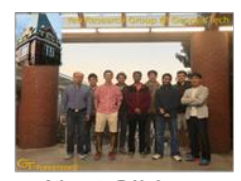

Next Slide

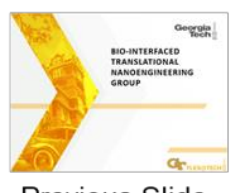

Previous Slide

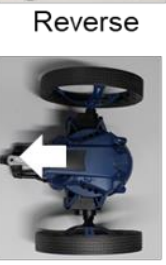

Reverse

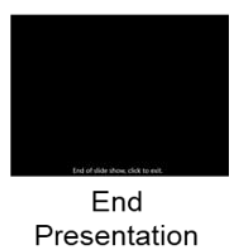

Figure 5. In vivo demonstration of SKINTRONICS BMI with human subjects. a-c, Subject seated in a powered wheelchair (a) with the LED stimulus array (b) and SKINTRONICS secured and conforming to the back of the neck (b). d, Android user interface during training and evaluation, showing time-domain data, corresponding power spectrum, and output class. e, EEG data recorded at each state, labeled as alpha rhythms, 11.1 Hz, 15.2 Hz, 12.5 Hz, 16.7 Hz SSVEP respectively. $\mathrm{f}-\mathrm{h}$, Three target machines to control via SSVEP signals, including a wireless electronic wheelchair (f) with 5 classes (no action, forward, rotate counterclockwise, rotate clockwise, and reverse, and wireless vehicle (g) with the same commands as the wheelchair, and PowerPoint presentation (h) with 5-class actions (no action, begin presentation, next slide, previous slide, and end presentation, respectively). 
Table 1. Comparison of information transfer rate (ITR) between the SKINTRONICS and reported values.

\begin{tabular}{|c|c|c|c|c|c|c|}
\hline & Year & $\begin{array}{c}\text { Accuracy } \\
(\%)\end{array}$ & $\begin{array}{c}\text { Length } \\
\text { (sec) }\end{array}$ & $\begin{array}{l}\text { Number of } \\
\text { classes }\end{array}$ & $\begin{array}{c}\text { Number of } \\
\text { channels }\end{array}$ & $\begin{array}{c}\text { ITR } \\
\text { (bits/min) }\end{array}$ \\
\hline $\begin{array}{c}\text { This work } \\
\text { (SKINTRONICS) }\end{array}$ & 2019 & 94.54 & 0.94 & 5 & 2 & $122.1 \pm 3.53$ \\
\hline$\underset{30}{\text { Nakanishi et al. }}$ & 2018 & 89.83 & 0.8 & 40 & 9 & $\begin{array}{c}325.33 \pm \\
38.17\end{array}$ \\
\hline$\underset{44}{\text { Chen et al. }}$ & 2015 & 91.09 & 1 & 40 & 9 & $270.0 \pm 61.8$ \\
\hline Bevilacqua et al. & 2014 & 95.61 & 2 & 3 & 4 & $38.44 *$ \\
\hline $\begin{array}{c}\text { Kwak et al. } \\
31\end{array}$ & 2017 & 99.19 & 2 & 5 & 8 & $67.13^{*}$ \\
\hline $\begin{array}{c}\text { Volosyak et al. } \\
60\end{array}$ & 2011 & 96.79 & 2 & 5 & 8 & $\begin{array}{c}61.70 \pm \\
32.68 \\
\end{array}$ \\
\hline $\begin{array}{c}\text { Lin et al. } \\
18\end{array}$ & 2007 & 73.00 & 1.5 & 9 & 8 & $60.74 *$ \\
\hline Bin et al. & 2009 & 95.30 & 2 & 5 & 8 & $58.6 \pm 9.6$ \\
\hline$\underset{14}{\text { Wang et al. }}$ & 2010 & 97.20 & 3.08 & 16 & 10 & 72.2 \\
\hline
\end{tabular}

*Calculated values from the reported data. 